\title{
A COMPARATIVE STUDY OF IN VIVO EFFECT OF PERCUTANEOUS BONE MARROW INJECTION VS WITHOUT INJECTION ON UNION OF OPEN TIBIAL SHAFT FRACTURES WITH EXTERNAL FIXATOR .
}

KEY WORDS:

\section{Hrishikesh Desai*

Assistant professor SBKS Medical college, Vadodara.

Assistant professor SBKS Medical college, Vadodara.

Background : Open tibial fractures are notorious fractures because the open wound leaves us with limited options and means multiple operations with long intervals for the patient. The usual method of treatment of contaminated, late presenting and complicated fractures is a temporary external fixator followed by conversion into a costly definitive procedure once the wound has healed. The secondary definitive procedure also means extra cost in an already economically stressed patient.In our set up we have to often let patients leave with a plaster cast after removing the external fixator for economic constrains. Per cetaceous autologous bone marrow injection while the patient is on external fixator is one alternative that we have tried to deal with this problem hoping for a primary union without any costly intervention. Materials and methods : We had 42 cases of open tibial fractures which were treated with external fixator and per cutaneous bone marrow injection while 38 other cases which were used as control with only the external fixator and no injections. We included open fractures of only Gustilo Anderson type II,IIIA and B for our study. Study was conducted between period of June 2012 and December 2013 at SBKS medical college. Results : In group one, 34 of the 42 patients had a radiological union before 12 weeks while another 2 in 16 weeks. For group 2 , only 16 of the 38patients had radiological union at 12 weeks and no more at 16 weeks. The average time of union for group 1 was 10 weeks while of group 2 was 12 weeks .Function of the union cases of both the groups was similar in all aspects including distance of walking and ability to carry out daily activities. Conclusion : Per cutaneous autologous bone marrow injections are cheap, easily available and successful alternative to a secondary procedure for open tibial fractures on external fixators.

\section{INTRODUCTION}

Open tibial fractures are notorious fractures because the open wound leaves us with limited options and means multiple operations with long intervals for the patient. Amongst the options available primary nailing is a controversial option wit variable results but practiced in fresh , not so contaminated wounds, even up to Gustilo 3B. But the major problem in the subcontinent is that fresh fractures come in late often after poor initial management, are grossly contaminated and are beyond the stage of primary definitive management. For these fractures the usual practiced method is an external fixator followed by a definitive procedure after the wound has been addressed. For wound coverage various grafting techniques are advocated but principally all of them usually lead to a delay in definitive fixation or increased risk of infection if it is done early and a costly hospital stay. This often leads to a less preferred but much practiced treatment of a plaster cast, following removal of the ex fix giving varying results which are never satisfactory. Thus we search for an alternative method to expedite the healing without a costly and time consuming secondary procedure which can be practiced while the patient is on the external fixator. Percutaneous autologous bone marrow injection is one such substitute that we have tried to use in this study.

\section{Materials and methods:}

We operated 104 cases of open fracture tibia with external fixator as their primary management within 2-48 hrs of presentation. The delay in presentation varied from 3 hours to 7 days with an average of 2.7 days. We included open fractures of only Gustilo Anderson type II,IIIA and B for our study. We included all diaphyseal fractures and diaphyseal fractures extending to metaphysic but excluded all intra articular extensions. Segmental fractures were also excluded from the study. The mean age of presentation was $26.6 \mathrm{yrs}$.

We started the study by creating 2 groups. The study group was treated secondarily with an injection of autologous bone marrow 0-2 1 days after the primary operation. We made it a point to give injections only after there was some kind of wound coverage so that the aspirate would not leak out of the open wound. The control group received no injections but all other treatment parameters remained the same.We assigned them to either of the groups on alternate days with no bias for the wound type or contamination. The patients continued receiving wound care management and repeat $\mathrm{x}$ - rays were done at 4,8,12 weeks and every 3 months after that. Knee bending exercises were given as soon as the patient was able to tolerate it but restricted if the tissue grafting procedure required so. Partial weight bearing was started at 4 weeks. Radiological union and limb function in the form of range of moment and later by walking and ability to perform daily activities.

Once radiological union was noticed the patient was classified as a success and external fixator was removed. Protected weight bearing (PTB cast with heel) was given to the patient and rehabilitated to unprotected weight bearing in a span of 6 weeks. Patients were encouraged to return to their daily activities and previous jobs during the rehabilitative phase. If even after 12 weeks no signs of radiological union were seen then the patient was classified as a failure. In these cases the external fixator was removed and a secondary procedure done to achieve union. If there was a doubt over union then a repeat $\mathrm{x}$ - ray was done after 4 weeks to classify them into either of the groups.

At the end of 1 and half year 3 patients succumbed to the other major injuries, 12 were lost to follow up, while 3 had an amputation due to infection or bleeding which could not be controlled, while 9 had a change of treatment plan. Of these 7 were due to uncontrolled pin tract infection while 2 due to patient's wish. We have not included any of these in our study since they would complicate the results. 42 of the rest of the 80 cases belonged to group 1 while 38 to group 2. The time of primary management and age of patients were almost equal for both groups.

Surgical technique : Patients were operated under regional or local anaesthesia. In the supine position a 16 gauge bone 
marrow aspiration needle was tapped in the middle of iliac crest. We used a $50 \mathrm{ml}$ syringe with $5 \mathrm{ml}$ of heparin (diluted $1: 1000)$ to aspirate bone marrow from multiple punctures till we obtained $30 \mathrm{ml}$ of the aspirate. Multiple aspirations with a minimum of 3 sites was preferred because prolonged aspiration leads to more of blood than progenitor cells in the aspirate. Also the heparin gives us extra time to aspirate before the aspirate clots. We did not use any concentration techniques. The aspirate was then simply injected at the site of fracture with or without image intensification. The whole procedure takes about 20 minutes and can be done on an outdoor basis even without an image intensifier.

\section{Results}

In group one, 34 of the 42 patients had a radiological union before 12 weeks while another 2 in 16 weeks. For group 2 , only 16 of the 38 patients had radiological union at 12 weeks and no more at 16 weeks. The average time of union for group 1 was 10 weeks while of group 2 was 12 weeks.

Function of the success cases of both the groups was similar in all aspects including distance of walking and ability to carry out daily activities. 12 Patients of the lst group complained of mild discomfort or pain in the iliac crest region after the procedure which subsided in a weeks time. One of them complained of a reddish swelling with pain and serosanguinous discharge which subsided after a course of oral antibiotics.

We did a detailed analysis of the 10 cases that were a failure in our study group (table) based on age, sex, delay in presentation and type of injury and time of injection. The most significant finding was that 8 of the 10 failure cases were given the injection after 3 days of the primary management. As expected majority of these cases were type IIIB injuries (7 out of 10) and average time of presentation of these 10 cases was more than that of the success cases(4.25 days and 2.5 days respectively). Age and sex has no consequence on the results. Infection control was not included in this study as all infected cases were excluded from this study. But retrospectively 2 out of the 3 amputations and 5 out of the 7 uncontrolled pin tract infections were in group 2 proving clearly the role of bone marrow injection in controlling infection also.

\begin{tabular}{|c|c|c|c|c|c|}
\hline $\begin{array}{c}\text { Failure } \\
\text { case no. }\end{array}$ & Age & Sex & $\begin{array}{c}\text { Delay in } \\
\text { presentation }\end{array}$ & Injury type & $\begin{array}{c}\text { Time of } \\
\text { injection }\end{array}$ \\
\hline l & 28 & M & 2 days & III A & 14 days \\
\hline 2 & 42 & M & 3 days & III B & 21 days \\
\hline 3 & 21 & F & 1 day & III B & 10 days \\
\hline 4 & 16 & M & 12 hours & III B & 14 days \\
\hline 5 & 30 & M & 4 days & III B & 3 days \\
\hline 6 & 15 & M & 1 days & II & 8 days \\
\hline 7 & 44 & F & 5 days & III B & 21 days \\
\hline 8 & 22 & M & 2 days & III B & 0 day \\
\hline 9 & 36 & F & 7 days & IIIA & 8 days \\
\hline 10 & 23 & M & 4 hrs & III B & 18 days \\
\hline
\end{tabular}

\section{Discussion and conclusion}

After enjoying early fame, the popularity of external fixators declined to the extent that some termed it as "non union machines". Presently the concept of external fixators is much more evolved and they are primarily considered as temporary devices to tide over the acute trauma to the limb. They are used not only in open fractures but also closed fractures with soft tissue trauma evident in the form of oedema, blisters and hematomas. The primary aim of the external fixator is to provide a stable frame with minimum motion at fracture site so that the soft tissue envelope around it may heal. Once this happens, usually in a period 1-4 weeks, the external fixator is removed and a definitive fixations done. The soft tissue healing thus gained improves the results tremendously. This sounds good in theory but the problem arises when the soft tissues do not heal within this $1-3$ week time. This is especially true for countries like ours where we receive late presenting grossly contaminated wounds in overcrowded hospitals. This leads to prolonged external fixators with persistent infection and swellings escalating the cost of treatment for the patient. Many times the patient is not able to afford a secondary definitive operation and the only solution left is a plaster cast which invariably leads to restriction of the joints incorporated. So we searched for alternatives to attain union while the patient is on the external fixators, thus providing an all in one solution to all these problems. Well known methods like electrical stimulation and bone morphogenic proteins are too expensive and difficult for the patient to follow diligently.

Per cutaneous autologous bone marrow injections have been successfully used to help and expedite the fracture union since long. It has also been used in delayed unions and non unions to achieve union. It has two major advantages over conventional bone grafting. Apart from preventing donor site morbidity we also get the advantage of doing this with minimal invasive techniques. The minimally invasive part not only makes it more cosmetic and a minor procedure but also prevents it from disturbing the fracture milieu and disrupting the soft tissues and dispersing the factors of fracture healing. 